\title{
CANONICAL FORMS FOR SYMMETRIC LINEAR VECTOR FUNCTIONS IN PSEUDO-EUCLIDEAN SPACE*
}

\author{
BY \\ R. V. CHURCHILL
}

1. Introduction. It is our purpose to find orthogonal directions which are determined by the symmetric linear vector function in pseudo-euclidean four-space such that, when referred to these directions, the function is described by a minimum number of independent scalars. We use the canonical forms found in this way to examine the structure of this function.

Algebraically our problem is that of reducing a matrix with the symmetry properties shown in (2) below to canonical forms in which a minimum number of independent elements appear, under linear transformations which leave the quadratic form $-\left(x^{1}\right)^{2}+\left(x^{2}\right)^{2}+\left(x^{3}\right)^{2}+\left(x^{4}\right)^{2}$ invariant. Although geometric methods are used, the algebraic as well as the geometric form of the results is given at the end $(\$ 9)$.

Canonical forms for symmetric linear vector functions are also canonical forms for symmetric tensors of the second rank. The prominent rôle played by tensors of this type in physics where the space is pseudo-euclidean lends importance to the problem of determining the nature of canonical forms for these tensors. In fact this problem presented itself to the author in attempting to extend the results of a study $\dagger$ of the Riemann curvature tensor in $E_{4}$ to a pseudo-euclidean four-space. The extension depends upon the canonical forms for the once-contracted Riemann tensor, a tensor which is symmetric and of second rank. The present paper shows that these results can not be extended without many changes, because the symmetric tensor has a structure much more complex in pseudo-euclidean than in euclidean space.

Canonical forms are known for antisymmetric linear vector functions in pseudo-euclidean four-space $\ddagger$ These forms apply to antisymmetric tensors of the second rank, another group of important tensors in physics.

In $E_{n}$ a symmetric linear vector function takes its canonical form when it is referred to its $n$ mutually perpendicular invariable directions. $\$$ Algebraically the result is that a symmetric matrix $A$ is reducible under an orthogonal transformation with matrix $B$ to a canonical form $B A B^{-1}$ in which all ele-

\footnotetext{
* Presented to the Society, November 28, 1931; received by the editors April 15, 1932.

† R. V. Churchill, these Transactions, vol. 34 (1932), p. 126.

$\ddagger$ G. Y. Rainich, Electrodynamics in the general relativity theory, these Transactions, vol. 27 (1925), p. 111; also see p. 113 for references to the corresponding problem in $E_{4}$.

\$ See D. J. Struik, Grund $\approx$ üge der mehrdimensionalen Differentialgeometrie, 1922, p. 33, or a review of this case by R. V. Churchill, loc. cit., p. 139.
} 
ments not in the principal diagonal are zero. ${ }^{*}$ This same form is obtained in one of the cases in the present paper.

2. The function. The scalar product of two vectors $x$ and $y$ in our fourdimensional pseudo-euclidean bundle is given by

$$
x y=-x^{1} y^{1}+x^{2} y^{2}+x^{3} y^{3}+x^{4} y^{4}
$$

in terms of contravariant components referred to a pseudo-cartesian coördinate system. There are three kinds of vectors: those with positive, negative, and zero squares. By introducing the coördinate vectors $i=(1,0,0,0)$, $j=(0,1,0,0), k=(0,0,1,0), l=(0,0,0,1), x$ can be written

$$
x=x^{1} i+x^{2} j+x^{3} k+x^{4} l .
$$

The coördinate vectors are mutually perpendicular, that is, their scalar products vanish; their squares have the values

$$
i^{2}=-1, j^{2}=k^{2}=l^{2}=1 .
$$

If $\lambda$ and $\mu$ are arbitrary scalars the family of vectors $\lambda x$ is called the direction of $x$, and $\lambda x+\mu y$ the plane of $x$ and $y$. Our space has three types of planes: those containing no directions of zero square, like the $k, l$ plane, those containing two such directions, like the $i, j$ plane, and those containing only one such direction, like the $i+j, k$ plane. The third is called the singular type of plane.

A linear vector function $f(x)$ assigns a vector to its vector argument and has the linearity property

$f(x)$ is symmetric if

$$
f(\lambda x+\mu y)=\lambda f(x)+\mu f(y)
$$

$$
f(x) y=f(y) x .
$$

Our function is determined by the way it transforms the coördinate vectors. Let $f_{1}{ }^{n}(n=1,2,3,4)$ be the contravariant components of $f(i)$, and $f_{2}{ }^{n}, f_{3}{ }^{n}, f_{4}{ }^{n}$ the components of $f(j), f(k)$, and $f(l)$ respectively. For example,

$$
f(i)=f_{1}^{1} i+f_{1}^{2} j+f_{1}^{3} k+f_{1}^{4} l .
$$

When $x$ is written in terms of the coördinate vectors the expansion of $f(x)$ shows that the components of the vector $f(x)$ are

$$
\sum_{\alpha} f_{u}^{p} x^{\alpha} \quad(\alpha, p=1,2,3,4) .
$$

The numbers $f_{m}{ }^{n}$ are the mixed coefficients for the linear vector function; in-

* This result seems to deserve more prominence than it is given in algebra; it is not mentioned in some of our best known text books. 
cidentally, they are the mixed components of the symmetric second-rank tensor $f(x) y_{\text {. }}$

Since $f_{1}^{2}=f(i) j$ and $f_{2}^{1}=-f(j) i$, etc., the symmetry property (1) becomes $f_{m}^{1}=-f_{1}^{m}$ and $f_{m}^{n}=f_{n}^{m}(m, n=2,3,4)$, so the scheme of coefficients for $f(x)$ can be written

$$
\left\|f_{m}{ }^{n}\right\|=\left\|\begin{array}{cccc}
f_{1}^{1} & f_{1}^{2} & f_{1}^{3} & f_{1}^{4} \\
-f_{1}^{2} & f_{2}{ }^{2} & f_{2}^{3} & f_{2}^{4} \\
-f_{1}^{3} & f_{2}{ }^{3} & f_{3}{ }^{3} & f_{3}^{4} \\
-f_{1}^{4} & f_{2}{ }^{4} & f_{3}{ }^{4} & f_{4}^{4}
\end{array}\right\| .
$$

If $f(x)=\lambda x$ where $\lambda$ is a scalar, then $x$ belongs to an invariable direction of $f(x)$. Similarly if $f(x)=\lambda x+\mu y$ and $f(y)=\lambda^{\prime} x+\mu^{\prime} y$ the plane of $x$ and $y$ is invariable. It is known that every linear vector function in four-dimensional space has at least one invariable plane.* We use this fact as a working basis for finding canonical forms of $f(x)$. The problem is divided into two cases according to the types of the invariable planes.

3. Case I, in general. Suppose $f(x)$ has an invariable plane of a nonsingular type, a plane containing either two or no directions of zero square.

If there is an invariable plane containing no directions of zero square then coördinate axes can be found so that the new $k$ and $l$ vectors lie in this plane. The new $i, j$ plane is also invariable, for $f(i) k=f(k) i=0$ because $f(k)=\lambda k+\mu l$; similarly $f(i) l=0$ and $f(j) k=f(j) l=0$ so $f(i)$ and $f(j)$ are perpendicular to the $k, l$ plane and hence they lie in the $i, j$ plane.

If $f(x)$ has an invariable plane containing two directions of zero square the new $i, j$ vectors can be made to fall in this plane. Then by using the symmetry property in the same way as before we find that the new $k, l$ plane is invariable, so this case reduces to the one just considered.

The invariable $k, l$ plane is an $E_{2}$ to which $f(x)$ is confined when $x$ is a vector in this plane; hence $f(x)$ has at least two perpendicular invariable directions in this plane. $\dagger$

Let coördinates be transformed again so that the new $k$ and $l$ vectors fall along these invariable directions while $i$ and $j$ remain unchanged. If $\omega_{3}, \omega_{4}$ be the multipliers of $k, l$ our function is described by the equations

$$
f(i)=\alpha i+\beta j, \quad f(j)=\beta^{\prime} i+\gamma j, \quad f(k)=\omega_{3} k, \quad f(l)=\omega_{4} l,
$$

* G. Y. Rainich, loc. cit., p. 109.

$\dagger$ D. J. Struik, loc. cit. This is easily proved for $E_{2}$, however, by the method used below to examine the $i, j$ plane for invariable directions. 
where $\beta^{\prime}=-\beta$ since $f(i) j=f(j) i{ }^{*}$ We have now found new coördinate vectors which reduce the scheme of coefficients (2) to

$$
\left\|\begin{array}{rlll}
\alpha & \beta & 0 & 0 \\
-\beta & \gamma & 0 & 0 \\
0 & 0 & \omega_{3} & 0 \\
0 & 0 & 0 & \omega_{4}
\end{array}\right\|
$$

Let us examine the $i, j$ plane for new coördinate vectors which will reduce the number of independent scalars in the scheme

$$
\left\|\begin{array}{rr}
\alpha & \beta \\
-\beta & \gamma
\end{array}\right\|
$$

for $f(x)$ in this plane. A vector $x^{1} i+x^{2} j$ belongs to an invariable direction if there is a scalar $\omega$ for which

$$
f\left(x^{1} i+x^{2} j\right)=\omega\left(x^{1} i+x^{2} j\right) .
$$

According to (3) the scalar form of the last equation is

$$
x^{1}(\alpha-\omega)-x^{2} \beta=0, x^{1} \beta+x^{2}(\gamma-\omega)=0 .
$$

These equations have solutions other than $x^{1}=x^{2}=0$ if

$$
\omega^{2}-\omega(\alpha+\gamma)+\alpha \gamma+\beta^{2}=0 \text {. }
$$

Let $\omega_{1}, \omega_{2}$ be the roots of (6); then the discriminant is $\psi_{1}^{2}-4 \psi_{2}$ where

$$
\psi_{1}=\alpha+\gamma=\omega_{1}+\omega_{2}, \psi_{2}=\alpha \gamma+\beta^{2}=\omega_{1} \omega_{2} .
$$

Now in the $i, j$ plane the Lorentz transformation or rotation of axes can be written in the vector form

$$
i^{\prime}=i \sec \theta+j \tan \theta, j^{\prime}=i \tan \theta+j \sec \theta,
$$

or in scalar form by multiplying by $x$. Inversions of axes must be added to make the transformations complete. Under (8) the coefficients in (3) transform as follows:

$$
\begin{gathered}
\alpha^{\prime}=\sigma^{2} \alpha-2 \sigma \tau \beta-\tau^{2} \gamma, \beta^{\prime}=\sigma \tau(\gamma-\alpha)+\left(\sigma^{2}+\tau^{2}\right) \beta, \\
\gamma^{\prime}=\sigma^{2} \gamma+2 \sigma \tau \beta-\tau^{2} \alpha,
\end{gathered}
$$

where $\sigma$ is written for $\sec \theta$ and $\tau$ for $\tan \theta . \psi_{1}$ and $\psi_{2}$ are invariant under (8) and inversions; in fact they are invariant under the general Lorentz trans-

\footnotetext{
* When $\omega_{3}=\omega_{4}$ every direction in the $k, l$ plane is invariable; this is the only case in which there are more than two such directions.
} 
formations in our four-space since they represent the sum and product of two roots of the characteristic equation of $f(x)$.

4. Case I, when $\psi_{1}^{2}>4 \psi_{2}$. If $\psi_{1}^{2}>4 \psi_{2}$ then (6) has distinct real roots $\omega_{1}$, $\omega_{2}$ and the $i, j$ plane contains two invariable directions. Let $p, q$ be vectors along these directions, so that $f(p)=\omega_{1} p$ and $f(q)=\omega_{2} q$. Since $f(p) q=f(q) p$ it follows that

$$
f(p) q-f(q) p=\left(\omega_{1}-\omega_{2}\right) p q=0,
$$

and hence $p q=0$, so the directions are perpendicular to each other.

We can now select new $i, j$ vectors, using (8), so that they fall along these directions, and our scheme (5) reduces to

$$
\left\|\begin{array}{cc}
\omega_{1} & 0 \\
0 & \omega_{2}
\end{array}\right\|
$$

The form (4) reduces to our first canonical form with all elements not in the principal diagonal equal to zero, so $f(x)$ is described by the canonical form

$$
f(x)=\omega_{1} x^{1} i+\omega_{2} x^{2} j+\omega_{3} x^{3} k+\omega_{4} x^{4} l .
$$

In this case then $f(x)$ has four mutually perpendicular invariable directions and when referred to these directions it can be described in terms of four numbers in place of the original ten used in (2).

5. Case I, when $\psi_{1}{ }^{2}<4 \psi_{2}$. In this case (6) has imaginary roots and $f(x)$ has no invariable directions in the $i, j$ plane. According to (9), however, $\alpha^{\prime}$ can be made to vanish if

$$
\gamma \sin ^{2} \theta+2 \beta \sin \theta-\alpha=0 .
$$

Likewise $\gamma^{\prime}=0$ if

$$
\gamma+2 \beta \sin \theta-\alpha \sin ^{2} \theta=0 .
$$

Since $\psi_{1}^{2}<4 \psi_{2}$ the discriminant of each of these equations is positive; moreover it is impossible for either to have a root $\sin \theta=1$ or $\sin \theta=-1$. If $\sin \theta=s$ is a root of one of them then $1 / s$ is a root of the other when $s \neq 0$. If $s=0$ satisfies one, then the second root of this equation is such that $\sin ^{2} \theta>1$ while the only root of the other satisfies $\sin ^{2} \theta<1$. Hence there are just two real principal angles $\theta$ which make $\alpha^{\prime} \gamma^{\prime}=0$.

Suppose one of the coefficients $\alpha$ or $\gamma$, say $\alpha$, has been made to vanish. When (12) is written in terms of the new coefficients we find that there is no second angle which makes the coefficient $\alpha^{\prime}$ vanish, while there is just one angle which makes $\gamma^{\prime}$ vanish.

It follows that unique directions for the coördinate axes can be found for which $\alpha^{\prime}=0$, and also unique directions can be found for which $\gamma^{\prime}=0$. By 
taking new $i, j$ vectors along one of these pairs of directions, and noting that the coefficients become equal to the invariants $\psi_{1}$ and $\left(\psi_{2}\right)^{1 / 2}$, the scheme (5) can be reduced at pleasure to one of the two canonical forms

$$
\left\|\begin{array}{cc}
0 & \psi_{2}^{1 / 2} \\
-\psi_{2}^{1 / 2} & \psi_{1}
\end{array}\right\|,\left\|\begin{array}{cc}
\psi_{1} & \psi_{2}^{1 / 2} \\
-\psi_{2}^{1 / 2} & 0
\end{array}\right\|
$$

We have shown in this case that there are two directions in the $i, j$ plane, one of positive square and one of negative square, which $f(x)$ transforms into perpendicular directions. When $i$ or $j$ is taken along one of these directions $f(x)$ can be described, respectively, by the forms

$$
\begin{aligned}
& f(x)=\left(x^{1} j-x^{2} i\right) \psi_{2}^{1 / 2}+\psi_{1} x^{2} j+\omega_{3} x^{3} k+\omega_{4} x^{4} l, \\
& f(x)=\left(x^{1} j-x^{2} i\right) \psi_{2}^{1 / 2}+\psi_{1} x^{1} i+\omega_{3} x^{3} k+\omega_{4} x^{4} l .
\end{aligned}
$$

6. Case I, when $\psi_{1}^{2}=4 \psi_{2}$. In this case $\omega_{1}=\omega_{2}$. One new canonical form is needed together with the three already found. If $\beta=0$ the form (5) reduces to a special case of the canonical form (10), and since every direction in the $i, j$ plane is invariable with multiplier $\omega_{1}$ the same form is taken for every set of coördinate axes in this plane.

If $\beta \neq 0$ it follows from (12) and (13) that when $\alpha^{2}<\gamma^{2}, f(x)$ takes the first form of (14) with $\psi_{2}{ }^{1 / 2}=\psi_{1} / 2$; and when $\alpha^{2}>\gamma^{2}$ it takes the second form of (14). If $\beta \neq 0$ and $\alpha^{2}=\gamma^{2}$ then $\alpha+\gamma=0$ and $\omega_{1}=\omega_{2}=0$. The scheme (5) becomes

$$
\left\|\begin{array}{rr}
\alpha & \alpha \\
-\alpha & -\alpha
\end{array}\right\|
$$

and under the transformations (9) this can be changed only to the extent of replacing $\alpha$ by any number different from zero. In particular it can be put in the canonical form

$$
\left\|\begin{array}{rr|}
1 & 1 \\
-1 & -1
\end{array}\right\|
$$

Under (15) every vector in the $i, j$ plane transforms into the $i+j$ direction and $f(i+j)=0$.

7. Case II. Suppose $f(x)$ has no invariable plane of the non-singular type. Then it has at least one invariable plane containing just one direction of zero square.

We take the vectors $i+j$ and $k$ in this plane; ${ }^{*}$ then

$$
f(i+j)=\omega_{1}(i+j)+\epsilon k, f(k)=\lambda(i+j)+\mu k,
$$

\footnotetext{
* For the proof that this can be done, see G. Y. Rainich, loc. cit., p. 111.
} 
where $\omega_{1}, \epsilon, \lambda, \mu$ are scalars. But $\epsilon=0$ because $f(i+j) k=f(k)(i+j)$. Also due to the symmetry of $f(x)$ the $i+j, l$ plane, which is absolutely perpendicular to the $i+j, k$ plane, is invariable so that

$$
f(i+j)=\omega_{1}(i+j), f(k)=\lambda(i+j)+\mu k, f(l)=\delta(i+j)+\omega_{4} l .
$$

An examination of the $i+j, k$ plane shows that in addition to the direction of $i+j$ the direction of $\lambda(i+j)+\left(\mu-\omega_{1}\right) k$ is invariable, with multiplier $\mu$. If $\mu \neq \omega_{1}$ this direction has a positive square. Likewise in the $i+j, l$ plane the direction of $\delta(i+j)+\left(\omega_{4}-\omega_{1}\right) l$ is invariable with multiplier $\omega_{4}$, and its square is positive if $\omega_{4} \neq \omega_{1}$. The two directions thus found determine an invariable plane of the non-singular type, so the conditions of Case II are not satisfied unless $\mu=\omega_{1}$ or $\omega_{4}=\omega_{1}$, or both.

The first two cases not being essentially different, we take $\mu=\omega_{1}$ and treat $\mu=\omega_{1}=\omega_{4}$ as a special case; hence

$$
f(i+j)=\omega_{1}(i+j), f(k)=\lambda(i+j)+\omega_{1} k, f(l)=\delta(i+j)+\omega_{4} l .
$$

If $\omega_{4}=\omega_{1}$ the direction of $\delta k-\lambda l$ is invariable with multiplier $\omega_{4}$, and we have seen that if $\omega_{4} \neq \omega_{1}$ the direction of $\delta(i+j)+\left(\omega_{4}-\omega_{1}\right) l$ is invariable with multiplier $\omega_{4}$. Hence there is always a direction of positive square which is invariable with this multiplier. We take a new $l$ vector in this direction, and keep $i+j$ fixed, so that

$$
f(i+j)=\omega_{1}(i+j), f(l)=\omega_{4} l .
$$

In the three-space perpendicular to this new $l$ we select new vectors $i$, $j, k$ so that the sum of $i$ and $j$ is the vector $i+j$ in (16). Then $f(x)$ is determined by the equations

$$
\begin{array}{ll}
f(i)=\rho i+.\left(\rho-\omega_{1}\right) j-\eta k, & f(j)=\left(\omega_{1}-\rho\right) i+\left(2 \omega_{1}-\rho\right) j+\eta k \\
f(k)=\eta(i+j)+\omega_{1} k, & f(l)=\omega_{4} l .
\end{array}
$$

The coefficients here are reduced to the number shown with the aid of (16) and the symmetry property (1); also, the second coefficient in $f(k)$ is necessarily $\omega_{1}$ since the $i+j, k$ plane must have the direction of $i+j$ as its only invariable direction in order to satisfy the conditions of Case II.

We can simplify (17) by introducing the new function

$$
\phi(x)=f(x)-\omega_{1} x .
$$

$\phi(x)$ and $f(x)$ have the same invariable directions but different multipliers; they also have the same invariable planes. According to (17),

$$
\phi(i)=\zeta(i+j)-\eta k, \phi(j)=-\zeta(i+j)+\eta k, \phi(k)=\eta(i+j),
$$


where $\zeta=\rho-\omega_{1}$. The function $\phi(x)$ transforms every $x$ in this $i, j, k$ space into a vector in the $i+j, k$ plane, and every vector in this plane into the $i+j$ direction; it reduces vectors in this direction to zero. In this space the only invariable direction of $\phi(x)$ is that of $i+j$, and the only invariable plane is that of $i+j, k$.

Two perpendicular vectors can be found, however, which transform under $\phi(x)$ into vectors with a common direction perpendicular to both vectors, giving a transformation like (18) with $\zeta=0$. The first two of the vectors

$$
\begin{aligned}
m^{\prime} & =\left(\zeta^{2}+4 \eta^{2}\right) i+\zeta^{2} j-2 \zeta \eta k, \\
n^{\prime} & =2 \eta j+\zeta k, p^{\prime}=-\zeta(i+j)+2 \eta k
\end{aligned}
$$

transform in this way; that is, if $m, n, p$ are coördinate vectors taken along $m^{\prime}, n^{\prime}, p^{\prime}$, respectively, then

$$
\phi(m)=-\eta^{\prime} p, \phi(n)=\eta^{\prime} p, \phi(p)=\eta^{\prime}(m+n),
$$

where $m n=m p=n p=0,-m^{2}=n^{2}=p^{2}=1$, and

$$
\eta^{\prime}=2 \eta^{2} /\left(\zeta^{2}+4 \eta^{2}\right)^{1 / 2}
$$

When the Lorentz transformation (8) is applied to $m$ and $n$ the new coördinate vectors can be selected so that the coefficient $\eta^{\prime}$ in (19) transforms into any number except zero. Let $i, j$ be the new vectors in the $m, n$ plane for which this coefficient becomes unity and let $p$ be called $k$; then

$$
\phi(i)=-k, \phi(j)=\dot{k}, \phi(k)=i+j .
$$

It follows from (20) that the only vectors in this three-space for which $\phi(x) x=0$ are vectors in the planes of $i, j$ and $i+j, k$. Except for rotations in the $i, j$ plane the directions of the coördinate vectors in (20) are the only distinct mutually perpendicular directions for $\phi(x)$ such that two of them transform into the third. Hence the form (20), with coefficients unity, is unique.

Since $f(x)=\phi(x)+\omega_{1} x$ this coördinate system reduces $f(x)$ to the following canonical form:

$$
f(i)=\omega_{1} i-k, f(j)=\omega_{1} j+k, f(k)=i+j+\omega_{1} k, f(l)=\omega_{4} l,
$$

where $\omega_{1}, \omega_{4}$ are the multipliers of the invariable directions of $i+j, l$.

8. Geometric formulation of results. We have seen that in Case I $f(x)$ has at least two invariable directions of positive square, and in Case II it has one of positive and one of zero square. Thus we have proved the following 
THEOREM. Every symmetric linear vector function in pseudo-euclidean fourspace has at least two perpendicular invariable directions and at least one of its invariable directions has a positive square.

The multipliers of invariable directions are the real roots of the characteristic equation of $f(x)$. A classification of the forms for $f(x)$ for different cases of invariable directions is essentially a classification according to the roots of this equation.

If $f(x)$ has at least two invariable directions of non-zero square then the scheme of coefficients (2) for $f(x)$ can be reduced to the form (4). According to (10), (14) and (15), this form (4) can always be reduced to one of the canonical forms

(I)
(a) $\left\|\begin{array}{llll}\omega_{1} & 0 & 0 & 0 \\ 0 & \omega_{2} & 0 & 0 \\ 0 & 0 & \omega_{3} & 0 \\ 0 & 0 & 0 & \omega_{4}\end{array}\right\|$,
(b) $\left\|\begin{array}{cccc}0 & \psi_{2}^{1 / 2} & 0 & 0 \\ -\psi_{2}^{1 / 2} & \psi_{1} & 0 & 0 \\ 0 & 0 & \omega_{3} & 0 \\ 0 & 0 & 0 & \omega_{4}\end{array}\right\|$,
(c) $\left\|\begin{array}{cccc}\psi_{1} & \psi_{2}^{1 / 2} & 0 & 0 \\ -\psi_{2}^{1 / 2} & 0 & 0 & 0 \\ 0 & 0 & \omega_{3} & 0 \\ 0 & 0 & 0 & \omega_{4}\end{array}\right\|$,
(d) $\left\|\begin{array}{rrll}1 & 1 & 0 & 0 \\ -1 & -1 & 0 & 0 \\ 0 & 0 & \omega_{3} & 0 \\ 0 & 0 & 0 & \omega_{4}\end{array}\right\|$.

If $f(x)$ has only one invariable direction of non-zero square, then, according to (21), the scheme (2) can be reduced in a unique way to the canonical form

$$
\left\|\begin{array}{cccc}
\omega_{1} & 0 & -.1 & 0 \\
0 & \omega_{1} & 1 & 0 \\
1 & 1 & \omega_{1} & 0 \\
0 & 0 & 0 & \omega_{4}
\end{array}\right\| .
$$

Except when there are planes or spaces of invariable directions (equal $\omega$ 's in (I)), $f(x)$ has either just two invariable directions both of positive square and it takes the form (Ib) or (Ic), or just one of positive and one of zero square (form (II)), or just two of positive and one of zero square (form (Id)), or three of positive and one of negative square (form (Ia)).

$f(x)$ can always be reduced to one of the five forms (I), (II). The axes which reduce $f(x)$ to one of these forms are uniquely determined except when equal multipliers $\omega$ appear in (I), in which case there is a family of coördinate systems any one of which can be used. 
By methods similar to those used above it can be shown that the set (I) can be replaced by an alternate set
(a) $\left\|\begin{array}{llll}\omega_{1} & 0 & 0 & 0 \\ 0 & \omega_{1} & 0 & 0 \\ 0 & 0 & \omega_{3} & 0 \\ 0 & 0 & 0 & \omega_{4}\end{array}\right\|$,
(b) $\left\|\begin{array}{rlll}\mu & \mu & 0 & 0 \\ -\mu & \lambda & 0 & 0 \\ 0 & 0 & \omega_{3} & 0 \\ 0 & 0 & 0 & \omega_{4}\end{array}\right\|$,
(c) $\left\|\begin{array}{rlll}\lambda & \mu & 0 & 0 \\ -\mu & \mu & 0 & 0 \\ 0 & 0 & \omega_{3} & 0 \\ 0 & 0 & 0 & \omega_{4}\end{array}\right\|$
(d) $\left\|\begin{array}{cccc}0 & \psi_{2}^{1 / 2} & 0 & 0 \\ -\psi_{2}^{1 / 2} & 0 & 0 & 0 \\ 0 & 0 & \omega_{3} & 0 \\ 0 & 0 & 0 & \omega_{4}\end{array}\right\|$,

so that, in Case I, $f(x)$ can always be reduced to one of these forms. But when $\lambda$ and $\mu$ are defined in terms of invariants it must be understood that $\mu=\psi_{2} / \psi_{1}$ and $\lambda=\psi_{1}-\psi_{2} / \psi_{1}$ except when $\psi_{1}=0$. If $\psi_{1}=0, \psi_{2} \neq 0$, then (I'd) is to be used. If $\psi_{1}=\psi_{2}=0$ either (I'a) with $\omega_{1}=0$ or else (Id), which is (I'b) with $-\lambda=\mu=1$, must be used; since there is no choice here it is necessary to add (Id) to the set $\left(I^{\prime}\right)$ when the latter is given in terms of invariants.

9. Algebraic formulation of results. To give the results in terms of matrices let $F$ be the matrix (2) and let $A$ be the matrix of the Lorentz transformation:

$$
X^{i}=\sum_{j} a_{j}{ }^{i} x^{j}(i, j=1,2,3,4) \text { with }-\left(x^{1}\right)^{2}+\left(x^{2}\right)^{2}+\left(x^{3}\right)^{2}+\left(x^{4}\right)^{2} \text { invariant }
$$

For every $F$ there is either an $A$ such that $A F A^{-1}$ takes the form (4) or an $A$ for which it takes the form (II). If it takes the form (4) there is also an $A$ which makes $A F A^{-1}$ take some one of the forms (I), and an $A$ which makes it take some one of the forms $\left(\mathrm{I}^{\prime}\right)$. The literal elements in (I) and (II) are values of invariants of $F$ under the transformations $A F A^{-1}$.

To state our results in terms of quadratic forms it is only necessary to note that we can write $f(x) x=\sum_{i j} f_{i j} x^{i} x^{j}$ where $f_{i j}=f_{i}{ }^{j}$ if $j \neq 1, f_{i 1}=-f_{i}^{1}$, and $f_{i j}=f_{j i}$.

Given two quadratic forms $\sum_{i j} f_{i j} \eta_{i} \eta_{j}$ and $\sum_{i j} g_{i j} \eta_{i} \eta_{j}(i, j=1,2,3,4)$ where the form $g$ has signature 2 . There is a real non-singular linear transformation of the $\eta$ 's which reduces $g$ to the form

$$
-\eta_{1}{ }^{2}+\eta_{2}^{2}+\eta_{3}{ }^{2}+\eta_{4}{ }^{2} \text {. }
$$

According to our results it is always possible to follow this transformation by a second which will leave the form (22) invariant and reduce the form $f$ to some 
one of the five forms

$$
\begin{gathered}
-\omega_{1} \eta_{1}^{2}+\omega_{2} \eta_{2}^{2}+\omega_{3} \eta_{3}^{2}+\omega_{4} \eta_{4}^{2}, \quad 2 \psi_{2}^{1 / 2} \eta_{1} \eta_{2}+\psi_{1} \eta_{2}{ }^{2}+\omega_{3} \eta_{3}^{2}+\omega_{4} \eta_{4}{ }^{2} \\
-\psi_{1} \eta_{1}^{2}+2 \psi_{2}^{1 / 2} \eta_{1} \eta_{2}+\omega_{3} \eta_{3}^{2}+\omega_{4} \eta_{4}{ }^{2},-\eta_{1}^{2}+2 \eta_{1} \eta_{2}-\eta_{2}{ }^{2}+\omega_{3} \eta_{3}{ }^{2}+\omega_{4} \eta_{4}{ }^{2} \\
\omega_{1}\left(-\eta_{1}{ }^{2}+\eta_{2}{ }^{2}+\eta_{3}{ }^{2}\right)-2 \eta_{1} \eta_{3}+2 \eta_{2} \eta_{3}+\omega_{4} \eta_{4}{ }^{2} .
\end{gathered}
$$

The coefficients here were read from the five forms (I), (II) after changing the signs of the elements in the first column of each matrix in (I), (II). Here, as before, the alternate forms $\left(I^{\prime}\right)$ can be used in place of $(I)$.

University of Michigan, ANn Arbor, Mich. 